\title{
The Mini Labyrinth - a Simple Benchmark for Radiation Protection and Shielding Analysis
}

\author{
Branislav Vrban ${ }^{1}$, Štefan Čerba ${ }^{1}$, Filip Osuský ${ }^{1}$, Jakub Lüley $^{1}$, Vladimír Nečas ${ }^{1}$, Karel Katovský ${ }^{2}$, \\ Ondrej Štastný ${ }^{2}$, Marko Gloginjić3 ${ }^{3}$, Marko Erich $^{3}$, Željko Mravik ${ }^{3}$, Srdjan Petrović3 \\ ${ }^{1}$ Slovak University of Technology in Bratislava, Institute of Nuclear and Physical Engineering, \\ Slovakia \\ ${ }^{2}$ Brno University of Technology, Department of Electrical Power Engineering, Czech Republic \\ ${ }^{3}$ Vinča Institute of Nuclear Sciences Laboratory of Physics, Serbia \\ branislav.vrban@stuba.sk
}

\begin{abstract}
The Mini Labyrinth experiment is a simple neutron and gamma shielding experiment developed at STU, inspired by the ALARM-CF-AIR-LAB-001 ICSBEP benchmark experiment. The STU Mini Labyrinth is approximately ten times smaller and consists of NEUTRONSTOP shielding blocks. This paper describes the second version of the Mini Labyrinth experiment and presents the results of the neutron and gamma fields simulation and measurement. The PuBe neutron source with the emission rate of $1.0 \mathrm{E} 7 \mathrm{n} . \mathrm{s}^{-1}$ was utilized in the experiment. The measurement of gamma ambient dose equivalent $H^{*}(10)$ and neutron count rates is performed by the Thermo Scientific RadEye portable survey meter. The simulation part was carried out using the state-of-the-art MCNP6 and SCALE6 MONACO stochastic calculation tools taking into account the detailed geometry of the labyrinth and a combined neutron and gamma source of particles. The comparisons were performed between codes and an experiment, based on the dose rate in the unique detection positions and using a $2 \mathrm{D}$ map of neutron and photon fluxes. The propagation of cross-section uncertainties was investigated through the shielding analysis. Partial agreement between codes and measurement was achieved, however serious discrepancies near the PuBe source were identified.
\end{abstract}

Keywords - radiation shielding, experiment, calculation, crosssection uncertainties, labyrinth.

\section{INTRODUCTION}

INCE World War II there has been significant development of methods and approaches used in the calculation of radiation shielding. This development was directly supported by the needs of industry (military technology, nuclear power plants, food processing, medical applications, accelerators, etc.). Over time, modeling and simulation of relevant effects shifted from analytical modeling to methods based on the socalled primary principles and their stochastic nature. Even nowadays it is necessary to know the accuracy of available computation codes, used nuclear data and it is desirable to evaluate the influence of the user on the final calculated parameter. One of the most effective ways of gaining user experience and minimizing user effects on the results of calculation is international collaboration comprising the designing and construction of relevant benchmark experiments. The construction phase is usually followed by simulation with tools available at the workplace (engineering tools vs. highfidelity methods), comparison of workgroup results and subsequent identification of the source of observed deviations from the experiment. Thus, this paper comprises a definition of the simple neutron and gamma shielding benchmark, inspired by the ALARM-CF-AIR-LAB-001 ICSBEP experiment [1]. The purpose of this experiment is to validate the computer codes of involved partners against real experimental data. In the following sections the measured quantities are compared to values calculated by MONACO (as a part of SCALE 6.2.4 system [2]) and MCNP 6 [3] stochastic codes. Moreover, the influence of different cross-section libraries and cross-section induced uncertainties are studied through the shielding analysis.

\section{EXPERIMENT SETUP}

Several experimental setups were created during the development of the experiment [4]. The Mini Labyrinth V02 version of the experiment with PuBe neutron source is utilized in this paper. The experimental setup comprises the $\mathrm{PuBe}$ neutron source with the emission rate of $1.0 \mathrm{E} 7 \mathrm{n} . \mathrm{s}^{-1}$, several NEUTRONSTOP $6 \times 12 \times 25 \mathrm{~cm}$ C-shape shielding blocks (polyethylene with $5 \%$ boron), $\mathrm{H}_{2} \mathrm{O}$ filled PLA tank, plastic source holder, and active and passive detectors. The STU Mini Labyrinth, is a mini version of the original ICSBEP Labyrinth, currently with dimensions of $96 \times 60 \times 25 \mathrm{~cm}$. The whole configuration is placed on a special desk, equipped with additional shielding material and gear. One of the main advantages of the experiment is the versatility in modifying the geometry configuration, the wall thickness, the height, and the width of the Mini Labyrinth. To allow a change of neutron spectra the graphite prism with a movable cavity for neutron source is placed on a desk near one of the entrances to the Mini Labyrinth. Due to the significant slowing down properties of graphite prism, it should be included in the simulation model even though it was not directly used in the specific experiment presented in the paper. The top view of the STU Mini Labyrinth is shown in Fig. 1. 


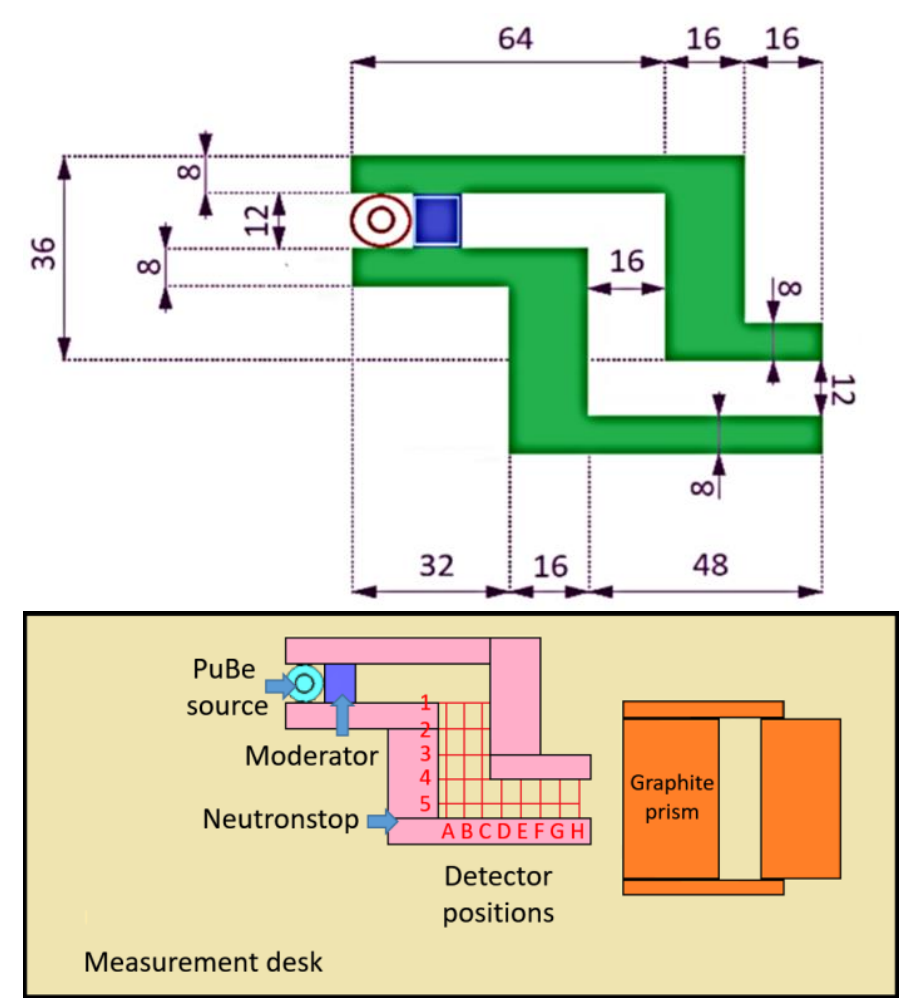

Fig. 1. STU Mini Labyrinth schematics (all dimensions are in $\mathrm{cm}$ ).

\section{Measurement Technique}

The measurements were carried out at the Mini Labyrinth experimental facility shown in Fig. 1. The whole measurement process consisted of the loading of the PuBe neutron source in the plastic holder placed behind the plastic tank filled with 2.4 liters of demineralized light water. Next, the measurement of neutron and gamma dose rates in 17 positions was carried out. The measurement positions were chosen on the $2 \mathrm{D}$ grid. The grid consists of 5 positions in the vertical direction (1-5) and 8 positions in the horizontal direction $(\mathrm{A}-\mathrm{H})$, while the first measurement position is referred to as $\mathrm{A} 1$ and the last one as H5. Each grid position (window) corresponds to the size of the detector base. However, due to detector dimensions, the fifth line was omitted in measurement, thus only $\mathrm{A} 1-\mathrm{H} 4$ positions were effectively used.

In each measurement position, the Thermo Scientific RadEyE SPRD-GN [5] digital dose meter was used. RadEye is a CLYC (Cesium Lithium Yttrium Chloride) scintillation detector calibrated to measure neutron and gamma count rate in units of CPS or CPM and ambient dose equivalent $\mathrm{H}^{*}(10)$ of gamma radiation in units of $\mu \mathrm{Sv} / \mathrm{h}$. The neutron measurement range of this detector is $0-1.0 \mathrm{E} 4 \mathrm{CPS}$ and the gamma measurement range is from $10 \mathrm{nSv} / \mathrm{h}$ to $250 \mu \mathrm{Sv} / \mathrm{h}$. It operates in two modes, "ratemeter" and "scaler".

At each position, the detector was placed in an upright position on the plastic holder ensuring that the crystal of the detector and the neutron source are in the same vertical position. $3 \times 20$ min measurement was performed, the ambient dose equivalent was obtained from the display and the neutron and gamma count rates were extracted from the RadEye Memory file. The final result per each position was evaluated as the average from the 3 measurements.

\section{Simulation}

\section{A. Radiation shielding}

The simulation part of this analysis was performed using two stochastic Monte Carlo calculation codes, MCNP6 and MONACO, which is part of the SCALE 6.2.4 package. For both codes, the detailed 3D model of the Mini Labyrinth was created. The PuBe neutron source was modeled as a metallic cylinder. The emission spectra of the source are shown in Fig. 2 and Fig. 3.

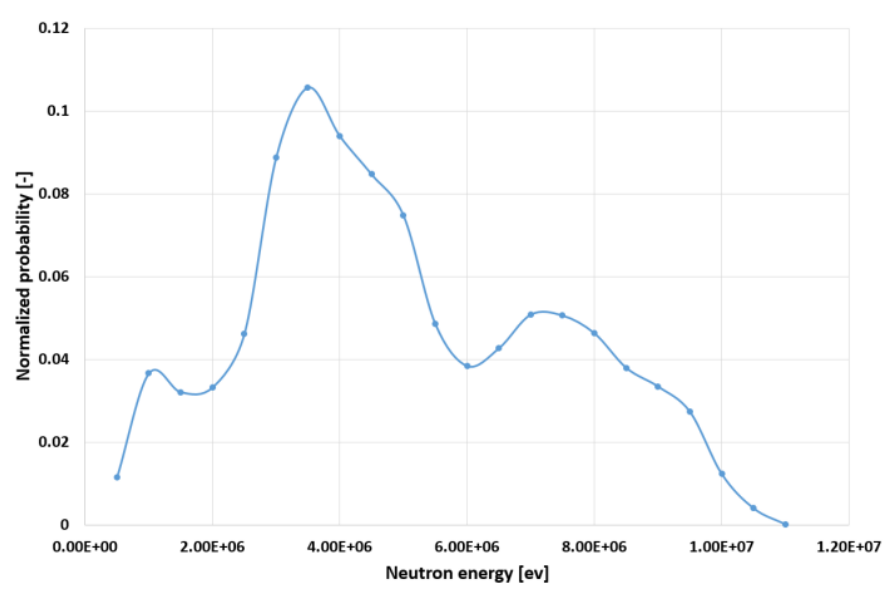

Fig. 2. PuBe neutron emission spectrum [6]

It should be noted, that the gamma emission spectra was modeled just as primary gammas from the $(n, \alpha)$ reaction. In this analysis, the angular distribution of the source was not considered.

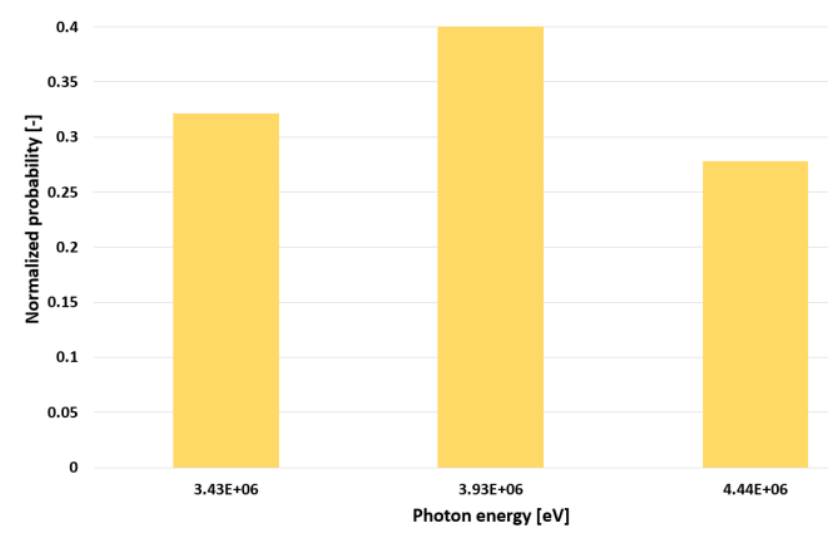

Fig. 3. PuBe photon emission spectrum

In both Monte Carlo codes, the calculations were performed as fixed source problems with the total number of 1.0E9 histories. In MCNP continuous energy cross-section libraries were used based on ENDF/B-VII.0 [7] and ENDF/B-VII.1 [8] evaluated data, processed by the STU staff. To estimate the gamma and neutron count rates, F5 type tallies were used both as mesh tallies and as point detectors in the four specified positions. The 
mesh tallies were modeled as a grid of $98 \times 98 \times 62 \times 1 \mathrm{~cm}$ parallelepiped elements. In MONACO the calculations were carried in forward mode, without using variance reduction techniques, and with standard $27 \mathrm{n} 19 \mathrm{~g}$ multi-group crosssection library, based on ENDF/B-VII.0 evaluated data. To allow comparison with MCNP, point detectors and mesh tallies were used in the calculations. The results were also multiplied by the source strength. To get comparable quantities with measurement the used detector flux-to-dose conversion factors were based on ICRU-57 [10] (ID 9036 for neutrons and ID 9510 for photons). The first response stands for the effective neutron dose and the second one represents the effective photon dose. The flux-to-dose conversion factors were chosen because of their ability to reasonably estimate the ambient dose equivalent $\mathrm{H}^{*}(10)$ for photons under $5 \mathrm{MeV}$ [11].

\section{B. Cross-section induced uncertainty}

The nuclear data used in a SCALE calculation are processed from evaluated nuclear data files $(\mathrm{ENDF} / \mathrm{B})$ produced by the National Nuclear Data Center through the AMPX code system. The ENDF/B data are based on a large set of nuclear data measurements, that have been evaluated by experts. Nuclear data are treated following the laws of probability and statistics. As such, they are represented by a central (recommended) value and are mostly burdened with uncertainty (in the form of covariance matrices) arising from measurements, modeling fits applied, or consistency adjustments. However, ENDF/B uncertainty data (including correlations) is not available for many nuclides or some type of data [11]. The present study aims to quantify nuclear data-induced uncertainties of the investigated quantities in the MONACO calculation explained above. To estimate the portion of simulation uncertainties induced by cross-section data, the stochastic procedure assuming that cross-section data used as an input to calculation may have a probability distribution, which is then propagated through a complex computational sequence. The SCALE system and super sequence SAMPLER with 200n47g crosssection library based on ENDF/B-VII.1 evaluated data is used in our approach. The SAMPLER module utilizes the previously generated perturbation factors for the 1D cross-sections on the multigroup library, assuming that the probability density functions are multivariate normal distributions with covariances given in the SCALE nuclear data covariance library. The SCALE 6.2 covariance library is based on ENDF/B-VII.1 data for 187 nuclides, combined with previous SCALE 6.1 covariances from other sources for another 215 nuclides [12]. Due to the stochastic approach applied, as the first attempt 40 independent calculations were performed. The evaluation of the final uncertainty in an individual requested output parameter $p$ was calculated by an auxiliary tool according to (1)

$$
\Delta p=\sqrt{\frac{1}{S-1} \sum_{s=1}^{S}\left(p_{s}^{M C}-\overline{p_{s}^{M C}}\right)^{2}},
$$

where $\Delta p$ is one standard deviation (1-sigma) of the investigated parameter due to uncertainties in the input parameters. The sign $p_{s}^{M C}$ stands for the result of the $s^{\text {th }}$ independent simulation (from the $S$ simulations in total) based on the Monte Carlo sampled input parameters which have been randomly varied within the specified distribution. In the calculation, the A1 detector calculation was assumed.

\section{RESUlts}

\section{A. Comparison of the simulated values}

The neutron flux mesh tallies (the top view at the level of neutron source) calculated in the SCALE 6.2.4 MONACO and MCNP6 can be seen in Fig. 4 and Fig. 5. Their relative change, shown in Fig. 6, highlights deviations mainly in the close vicinity of the source and at the entrance corridor to the Labyrinth. Here the relative change reaches values between 50 to $91 \%$. Farther distances show smaller deviations about the level of $10 \% \mathrm{We}$ believe that these discrepancies are emerging from the different continuous energy and the multigroup approach utilized in MCNP and SCALE. The effect of the multigroup approach may be amplified by the use of just 27 neutron and 19 photon groups in the MONACO calculation.

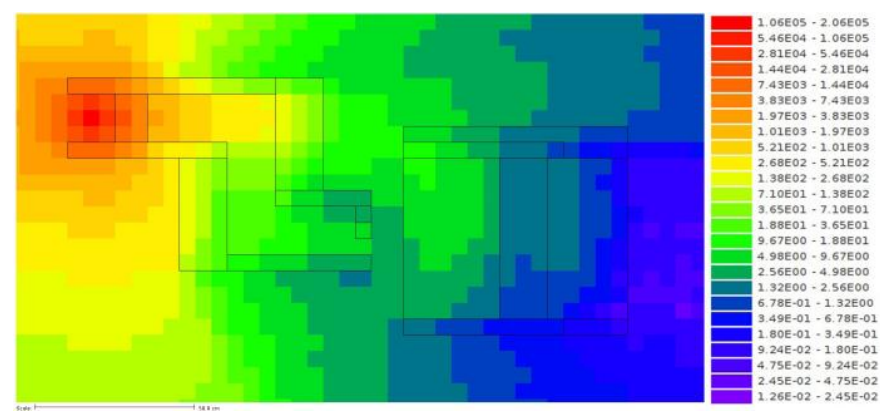

Fig. 4. Total neutron flux - SCALE $\left[\mathrm{par} . \mathrm{cm}^{-2} \cdot \mathrm{s}^{-1}\right]$

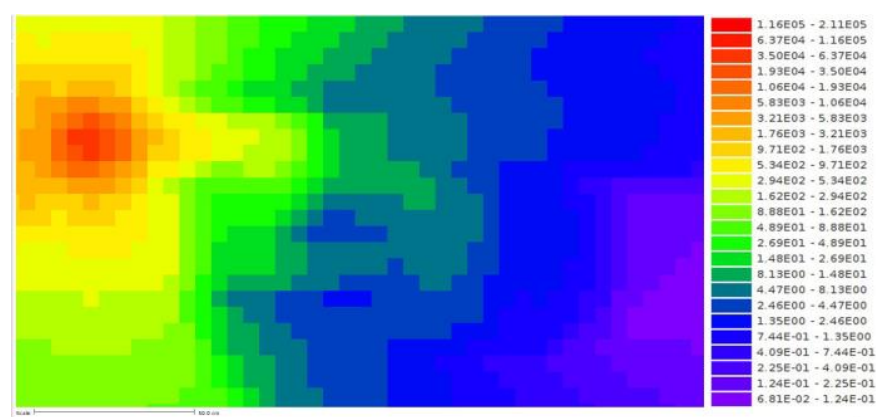

Fig. 5. Total neutron flux - MCNP $\left[\right.$ par.cm- $\left.{ }^{-2} . \mathrm{s}^{-1}\right]$

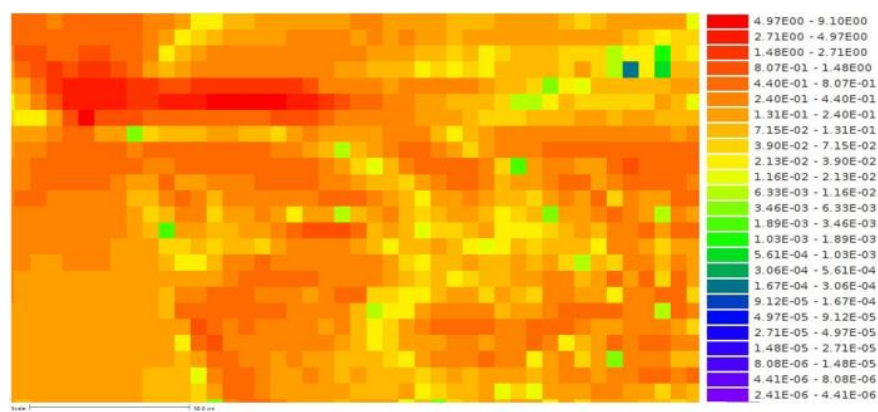

Fig. 6. Relative change of total neutron flux (MCNP as reference) 
The gamma results in Fig. 7 to Fig. 9, show similar behavior. Nevertheless, the better gamma attenuation of the NEUTRONSTOP blocks in MCNP can be observed. The relative changes are lower than in the case of neutrons, however, the localization of the biggest discrepancies perfectly match. Again, we believe that the source of discrepancies relies in the different energy structures used within both codes.

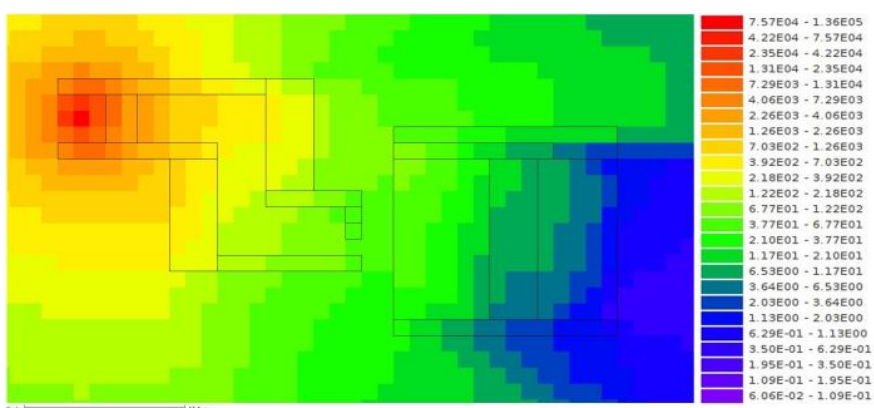

Fig. 7. Total photon flux - SCALE $\left[\right.$ par.cm $\left.{ }^{-2} \cdot \mathrm{s}^{-1}\right]$

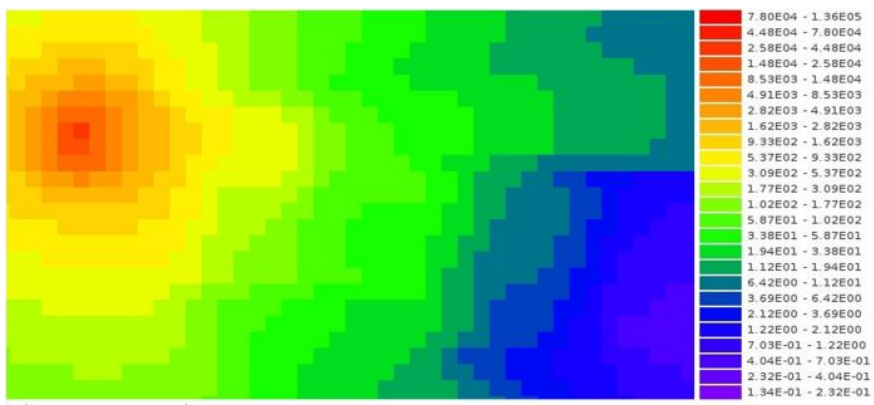

Fig. 8. Total photon flux - MCNP $\left[\right.$ par.cm $\left.{ }^{-2} . \mathrm{s}^{-1}\right]$

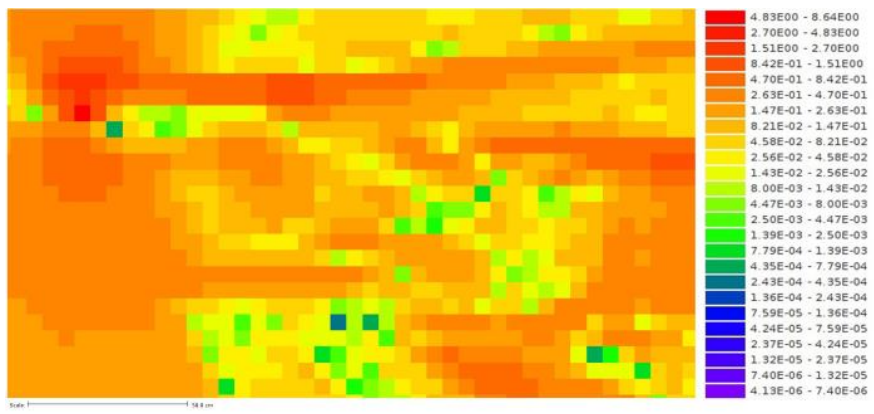

Fig. 9. Relative change of total photon flux (MCNP as reference)

The summary of calculated neutron and gamma fluxes in MCNP and SCALE for 17 detector positions can be found in Table I and Table II. The tables include both neutron and gamma fluxes, the statistical error resulting from the Monte Carlo calculation, the cross-section induced uncertainty determined in the next section, and the relative deviation from the MCNP results. Although the cross-section induced uncertainty was calculated in SCALE, as the first approximation, these values were applied also to MCNP.
TABLE I

CALCUlated NEUTRON FluXes

\begin{tabular}{lccc}
\hline \hline $\begin{array}{l}\text { Meas. } \\
\text { Pos. }\end{array}$ & $\begin{array}{c}\text { MCNP } \\
{\left[\mathrm{par} . \mathrm{cm}^{-2} . \mathrm{s}^{-1}\right]}\end{array}$ & $\begin{array}{c}\text { SCALE } \\
{\left[\mathrm{par} . \mathrm{cm}^{-2} . \mathrm{s}^{-1}\right]}\end{array}$ & $\Delta_{\mathrm{MCNP}}[\%]$ \\
\hline$A 1$ & $9.42 \mathrm{E}+01 \pm 1.81 \mathrm{E}+00$ & $1.03 \mathrm{E}+02 \pm 2.33 \mathrm{E}+00$ & $8.94 \pm 0.01$ \\
$A 2$ & $4.00 \mathrm{E}+01 \pm 7.93 \mathrm{E}-01$ & $4.46 \mathrm{E}+01 \pm 1.10 \mathrm{E}+00$ & $11.34 \pm 0.02$ \\
$A 3$ & $2.73 \mathrm{E}+01 \pm 5.52 \mathrm{E}-01$ & $3.01 \mathrm{E}+01 \pm 7.89 \mathrm{E}-01$ & $10.04 \pm 0.03$ \\
$A 4$ & $2.22 \mathrm{E}+01 \pm 4.55 \mathrm{E}-01$ & $2.42 \mathrm{E}+01 \pm 6.52 \mathrm{E}-01$ & $8.76 \pm 0.04$ \\
$B 1$ & $1.12 \mathrm{E}+02 \pm 2.14 \mathrm{E}+00$ & $1.19 \mathrm{E}+02 \pm 2.60 \mathrm{E}+00$ & $5.74 \pm 0.01$ \\
$B 2$ & $4.39 \mathrm{E}+01 \pm 8.70 \mathrm{E}-01$ & $4.77 \mathrm{E}+01 \pm 1.15 \mathrm{E}+00$ & $8.58 \pm 0.01$ \\
$B 3$ & $2.73 \mathrm{E}+01 \pm 5.52 \mathrm{E}-01$ & $2.98 \mathrm{E}+01 \pm 7.58 \mathrm{E}-01$ & $8.96 \pm 0.03$ \\
$B 4$ & $2.04 \mathrm{E}+01 \pm 4.21 \mathrm{E}-01$ & $2.24 \mathrm{E}+01 \pm 5.90 \mathrm{E}-01$ & $9.70 \pm 0.04$ \\
$C 1$ & $1.30 \mathrm{E}+02 \pm 2.47 \mathrm{E}+00$ & $1.34 \mathrm{E}+02 \pm 2.91 \mathrm{E}+00$ & $2.85 \pm 0.01$ \\
$C 2$ & $4.68 \mathrm{E}+01 \pm 9.22 \mathrm{E}-01$ & $5.02 \mathrm{E}+01 \pm 1.23 \mathrm{E}+00$ & $7.37 \pm 0.01$ \\
$C 3$ & $2.73 \mathrm{E}+01 \pm 5.52 \mathrm{E}-01$ & $3.00 \mathrm{E}+01 \pm 7.76 \mathrm{E}-01$ & $9.61 \pm 0.03$ \\
$C 4$ & $1.86 \mathrm{E}+01 \pm 3.86 \mathrm{E}-01$ & $2.11 \mathrm{E}+01 \pm 5.61 \mathrm{E}-01$ & $13.05 \pm 0.05$ \\
$D 4$ & $1.34 \mathrm{E}+01 \pm 2.83 \mathrm{E}-01$ & $1.44 \mathrm{E}+01 \pm 4.17 \mathrm{E}-01$ & $8.06 \pm 0.09$ \\
$E 4$ & $8.36 \mathrm{E}+00 \pm 1.84 \mathrm{E}-01$ & $9.33 \mathrm{E}+00 \pm 2.92 \mathrm{E}-01$ & $11.65 \pm 0.17$ \\
$F 4$ & $5.90 \mathrm{E}+00 \pm 1.35 \mathrm{E}-01$ & $6.60 \mathrm{E}+00 \pm 2.21 \mathrm{E}-01$ & $11.83 \pm 0.28$ \\
$G 4$ & $4.65 \mathrm{E}+00 \pm 1.09 \mathrm{E}-01$ & $5.49 \mathrm{E}+00 \pm 2.01 \mathrm{E}-01$ & $17.92 \pm 0.42$ \\
$H 4$ & $4.48 \mathrm{E}+00 \pm 1.05 \mathrm{E}-01$ & $5.20 \mathrm{E}+00 \pm 1.91 \mathrm{E}-01$ & $16.22 \pm 0.44$ \\
\hline \hline
\end{tabular}

TABLE II

CALCUlATED GAMMA FluXES

\begin{tabular}{lccc}
\hline \hline $\begin{array}{c}\text { Meas. } \\
\text { Pos. }\end{array}$ & $\begin{array}{c}\mathrm{MCNP} \\
{\left[\mathrm{par} . \mathrm{cm}^{-2} . \mathrm{s}^{-1}\right]}\end{array}$ & $\begin{array}{c}\text { SCALE } \\
{\left[\mathrm{par} . \mathrm{cm}^{-2} \cdot \mathrm{s}^{-1}\right]}\end{array}$ & $\Delta_{\mathrm{MCNP}}[\%]$ \\
\hline$A 1$ & $4.25 \mathrm{E}+02 \pm 2.65 \mathrm{E}+00$ & $4.21 \mathrm{E}+02 \pm 2.23 \mathrm{E}+00$ & $-1.04 \pm 0.01$ \\
$A 2$ & $3.10 \mathrm{E}+02 \pm 2.03 \mathrm{E}+00$ & $3.12 \mathrm{E}+02 \pm 1.73 \mathrm{E}+00$ & $0.80 \pm 0.01$ \\
$A 3$ & $2.56 \mathrm{E}+02 \pm 1.73 \mathrm{E}+00$ & $2.60 \mathrm{E}+02 \pm 1.47 \mathrm{E}+00$ & $1.68 \pm 0.01$ \\
$A 4$ & $2.19 \mathrm{E}+02 \pm 1.55 \mathrm{E}+00$ & $2.24 \mathrm{E}+02 \pm 1.28 \mathrm{E}+00$ & $2.18 \pm 0.01$ \\
$B 1$ & $3.82 \mathrm{E}+02 \pm 2.42 \mathrm{E}+00$ & $3.78 \mathrm{E}+02 \pm 1.96 \mathrm{E}+00$ & $-1.23 \pm 0.01$ \\
$B 2$ & $2.70 \mathrm{E}+02 \pm 1.83 \mathrm{E}+00$ & $2.71 \mathrm{E}+02 \pm 1.46 \mathrm{E}+00$ & $0.49 \pm 0.01$ \\
$B 3$ & $2.17 \mathrm{E}+02 \pm 1.52 \mathrm{E}+00$ & $2.21 \mathrm{E}+02 \pm 1.22 \mathrm{E}+00$ & $1.54 \pm 0.01$ \\
$B 4$ & $1.85 \mathrm{E}+02 \pm 1.34 \mathrm{E}+00$ & $1.89 \mathrm{E}+02 \pm 1.05 \mathrm{E}+00$ & $2.15 \pm 0.01$ \\
$C 1$ & $3.70 \mathrm{E}+02 \pm 2.37 \mathrm{E}+00$ & $3.65 \mathrm{E}+02 \pm 1.96 \mathrm{E}+00$ & $-1.30 \pm 0.01$ \\
$C 2$ & $2.56 \mathrm{E}+02 \pm 1.76 \mathrm{E}+00$ & $2.57 \mathrm{E}+02 \pm 1.41 \mathrm{E}+00$ & $0.66 \pm 0.01$ \\
$C 3$ & $1.99 \mathrm{E}+02 \pm 1.43 \mathrm{E}+00$ & $2.03 \mathrm{E}+02 \pm 1.15 \mathrm{E}+00$ & $2.15 \pm 0.01$ \\
$C 4$ & $1.60 \mathrm{E}+02 \pm 1.20 \mathrm{E}+00$ & $1.69 \mathrm{E}+02 \pm 9.45 \mathrm{E}-01$ & $5.27 \pm 0.01$ \\
$D 4$ & $1.38 \mathrm{E}+02 \pm 1.06 \mathrm{E}+00$ & $1.42 \mathrm{E}+02 \pm 8.23 \mathrm{E}-01$ & $3.14 \pm 0.01$ \\
$E 4$ & $1.12 \mathrm{E}+02 \pm 9.14 \mathrm{E}-01$ & $1.17 \mathrm{E}+02 \pm 7.06 \mathrm{E}-01$ & $4.46 \pm 0.01$ \\
$F 4$ & $8.50 \mathrm{E}+01 \pm 7.36 \mathrm{E}-01$ & $9.06 \mathrm{E}+01 \pm 5.73 \mathrm{E}-01$ & $6.53 \pm 0.01$ \\
$G 4$ & $6.60 \mathrm{E}+01 \pm 6.15 \mathrm{E}-01$ & $7.14 \mathrm{E}+01 \pm 4.62 \mathrm{E}-01$ & $8.09 \pm 0.01$ \\
$H 4$ & $4.91 \mathrm{E}+01 \pm 4.93 \mathrm{E}-01$ & $5.86 \mathrm{E}+01 \pm 4.08 \mathrm{E}-01$ & $19.27 \pm 0.02$ \\
\hline \hline
\end{tabular}

The comparison of the energy integrated neutron and gamma fluxes showed just partial agreement between the calculation tools. In terms of the total neutron flux, MONACO significantly overestimated the MCNP results with the average relative change from MCNP of $10.04 \%$. The maximal relative change is $17.92 \%$. The largest deviations are achieved in the case of detector positions $\mathrm{G} 4$ and $\mathrm{H} 4$, where the neutron flux values are the lowest. In the case of gammas, the average relative change is only $3.23 \%$ and the maximal error was $19.27 \%$, which is, again, achieved in measurement position $\mathrm{H} 4$. If we compare the gamma fluxes only for positions between $\mathrm{A} 1$ and $\mathrm{C} 3$, the relative error is only $1.11 \%$. Based on the partial results achieved, it can be concluded, that the results of both codes are showing the same trends, at detector positions may be considered partially acceptable, but cannot be judged as 
acceptable near the PuBe source and at the entrance corridor to the Labyrinth.

The comparisons of the neutron and gamma spectra per detector position calculated by MONACO are shown in Fig. 10 and Fig. 11. Each graph consists of data for 3 detector positions (A1, D4, and H4). In terms of the neutron count rate, and in accordance with our expectations, more slowed-down neutrons occurred at position $\mathrm{H} 4$ located farther from the source. In this position, the portion of thermal $(\mathrm{E}<1 \mathrm{eV})$ neutrons is $8.98 \%$. This is caused by the reflection of thermal neutrons from the graphite prism. This increase of thermal neutrons is compensated by a significant, $9.34 \%$ decrease of neutrons with energy above $100 \mathrm{keV}$. The hardest neutron spectrum was achieved in position D4, here the thermal neutron rate was only $6.11 \%$. This is caused by the absorption of thermal neutrons by the NEUTRONSTOP shielding blocks.

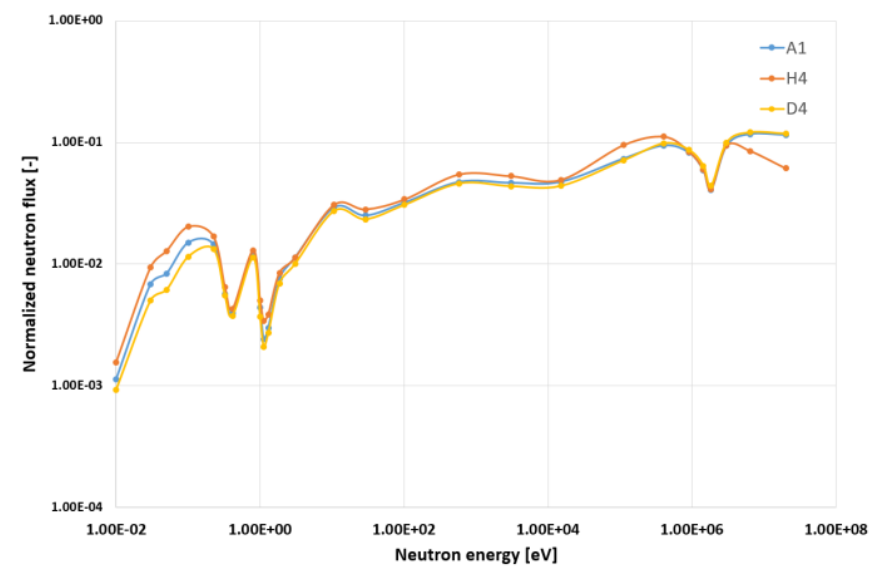

Fig. 10. Neutron spectra in A1, D4 and $\mathrm{H} 4$ positions

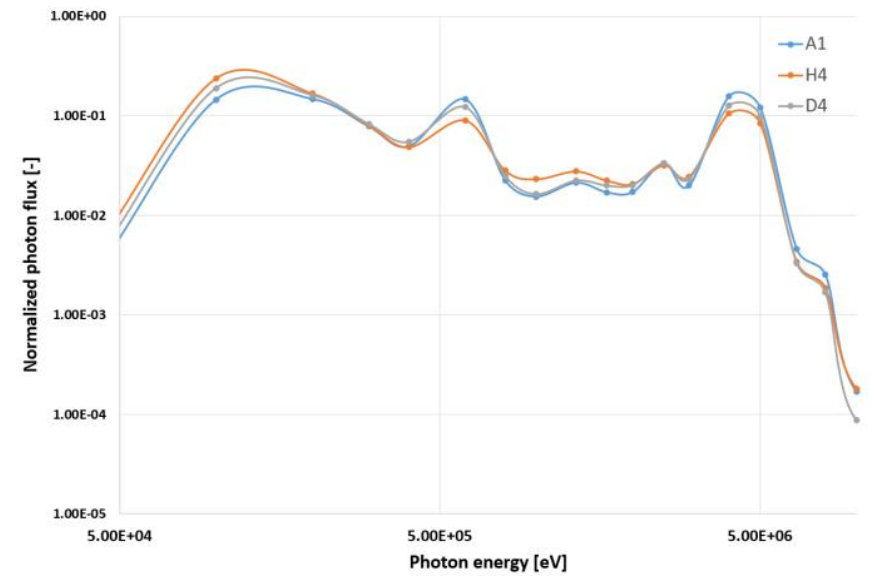

Fig. 11. Photon spectra in A1, D4 and H4 positions

The gamma spectra are showing considerably smaller deviations between detector positions. The largest discrepancies can be observed mainly in the energy intervals above $4 \mathrm{MeV}$ and below $400 \mathrm{keV}$. The relatively small difference of spectra (and thus low attenuation) is caused by the presence of mainly light nuclei $(\mathrm{H}, \mathrm{B}$, and $\mathrm{O})$ in the Mini Labyrinth materials.

\section{B. Results of the cross-section induced uncertainty}

The results presented in this section demonstrate the crosssection induced uncertainties to the effective doses from neutrons and photons. As illustrated in Fig. 12 and Fig. 13, the one sigma range of cross-section induced uncertainty to the effective dose from neutrons is $2.74 \%$, while the one sigma range of uncertainty to effective dose from photons of $0.62 \%$ is considerably smaller.

XS induced uncertainty: Neutron Point Detector - +/- 1 sigma

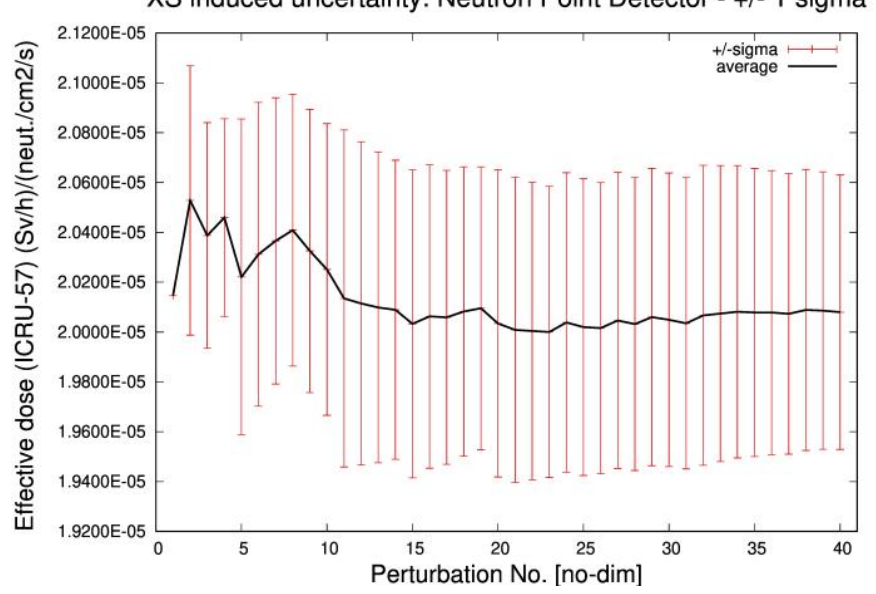

Fig. 12. Converge plot of the mean neutron effective dose and its uncertainty

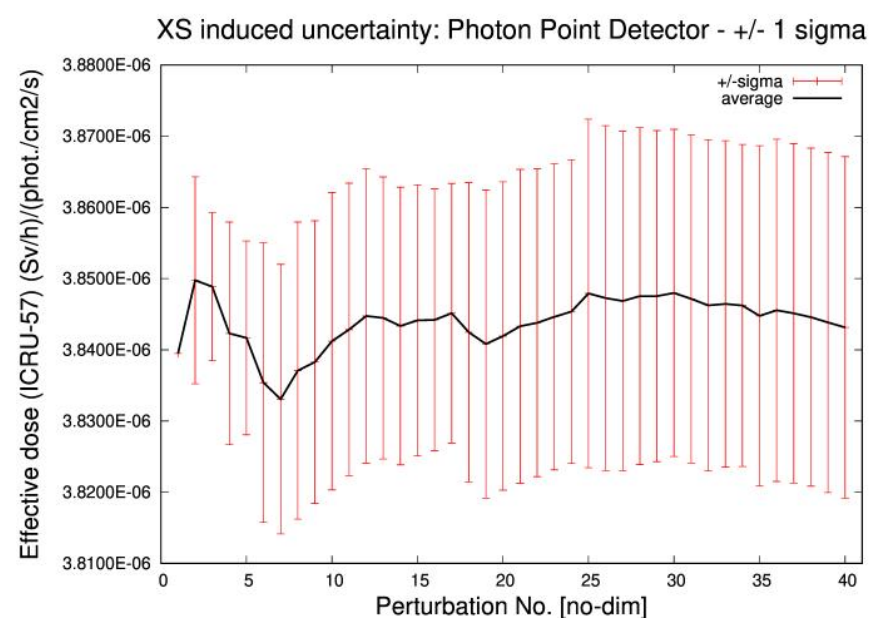

Fig. 13. Converge plot of the mean gamma effective dose and its uncertainty

The Monte Carlo uncertainty of the simulation was not taken into account due to its uniformity between cases and due to its size, which is by two orders of magnitude smaller than the cross-section induced uncertainty itself.

Interestingly, due to the energy dependence of flux-to-dose factors, the cross-section induced uncertainties to the integral neutron and photon fluxes are approx. 1.5 times smaller than in the case of effective doses. Based on the checked convergence plots, 40 independent calculations appear to be sufficient to obtain the first acceptable results. Anyhow, more perturbations are going to be calculated in near future. 


\section{Comparison of the simulation with measurements}

The comparison of the measured and simulated neutron count rates is shown in Fig. 14. The figure includes the results from both calculation codes and the RadEye detector. It should be noted that the presented values are normalized in the sense that the measured count rate in the case of RadEye and the neutron fluxes in the case of the simulations are divided by the sum of count rates or fluxes per each detection position. It should be also noted, that while in the case of MCNP and SCALE, the uncertainty represents the statistical uncertainty of the Monte Carlo game plus the cross-section induced uncertainties, in the case of the RadEye detector it represents the mean squared error of the measured values.

As it can be seen from the Fig. 14, for some measurement positions, such as $\mathrm{A} 4, \mathrm{~B} 4, \mathrm{C} 3$, or $\mathrm{C} 4$, the agreement between calculation and simulation is satisfactory, but for the rest of the positions, the results are not convincing. The most significant deviations were achieved for positions A1, B1, and C1, which are close to the wall of the Mini Labyrinth, and for positions D4 - H4, which are very far from the source. These deviations can be attributed to the spectral effect (and thus to the energy response function of the detector) of scattered neutrons on the wall of the mini labyrinth and also the possible evaporation of light water moderator during the measurement.

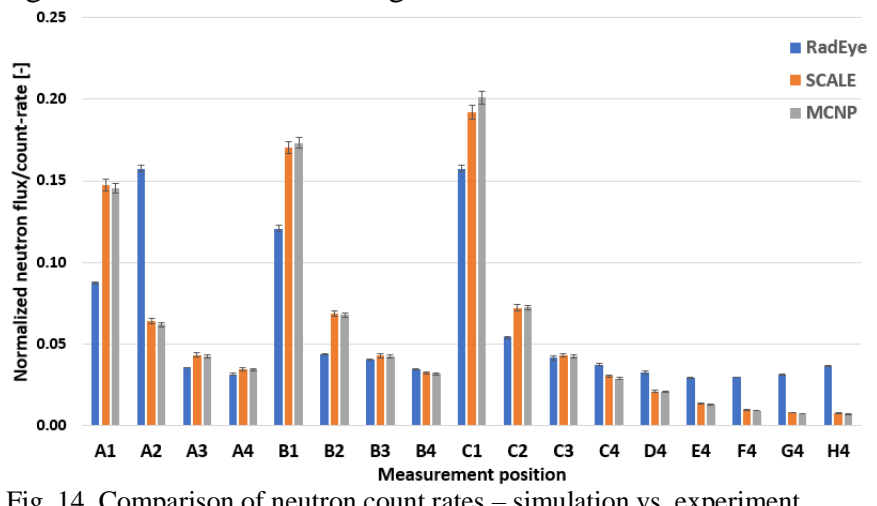

Fig. 14. Comparison of neutron count rates - simulation vs. experiment

The comparison of the measured ambient dose equivalent $\mathrm{H}^{*}(10)$ rates and effective dose rates calculated by the SCALE system is presented in Fig. 15. The comparison showed an average relative change of $6.65 \%$ with the maximal relative change of $\pm 16 \%$. The largest discrepancies were achieved for positions A4, A3, B4, D4, and H4 measurement positions.

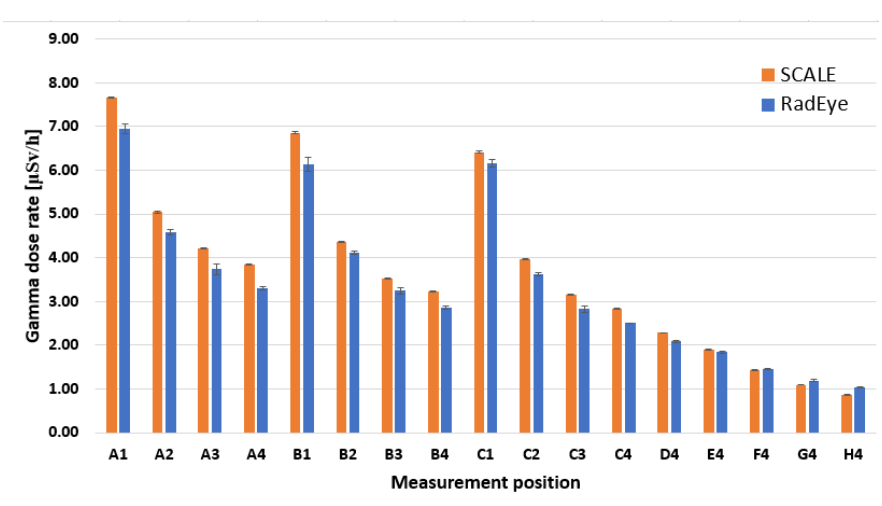

Fig. 15. Comparison of photon effective dose (SCALE) and measured $\mathrm{H}^{*}(10)$
Considering the slightly different units compared and the coarse energy group structure used in the calculation, we judge these results as acceptable. In the future $\mathrm{H}^{*}(10)$ flux-to-dose factors will be used in simulation to be able to achieve more credible results. Moreover, to minimize the relative change between the calculations and measurements, the source definition should be thoroughly reviewed.

\section{CONCLUSIONS}

The paper describes the Mini Labyrinth experiment developed at STU in Bratislava designed to assess the performance of computed codes in neutron and photon transport simulations. The first simulation results achieved by SCALE and MCNP show the discrepancies mainly at the entrance corridor to the Labyrinth and near the graphite prism. The results at detector positions are partially acceptable. We assume, that these discrepancies are emerging from the different continuous energy and the multigroup approach utilized in both codes. The effect of the multigroup approach may be amplified by the use of just 27 neutron and 19 photon groups in the MONACO calculation. In the future, also finer energy structures will be investigated. To partially understand the uncertainties included in the simulations, the cross-section induced uncertainties of effective doses from neutrons and photons were determined by the SAMPLER supersequence. The determined one sigma range of cross-section induced uncertainty to the effective dose from neutrons is $2.74 \%$, while the one sigma range of uncertainty to effective dose from photons is $0.62 \%$. These values may be considered in further designs of radiation shielding protection equipment and in case of comparison with measured values. The comparison of the measured and simulated neutron count rates is not convincing. Some measurement positions are in good agreement with simulations (A4, B4, C3, and C4) others are not satisfactory. These deviations have to be further investigated, but can be attributed to the spectral effect and the unknown energy response function of the detector used in the measurement. The comparison of the measured ambient dose equivalent $\mathrm{H}^{*}(10)$ and effective dose calculated by the SCALE system showed acceptable agreement. In the future $\mathrm{H}^{*}(10)$ flux-to-dose factors will be used in simulation to be able to achieve more credible results. The presented results and approaches serve us as a stepping stone for our further investigations and improvement of the Mini Labyrinth experiment at STU.

\section{ACKNOWLEDGMENT}

This study has partially been financially supported by the Slovak Research Development Agency No. APVV DS-FR-190014, No. APVV 16-0288 projects and by the Scientific Grant Agency of the Ministry of Education of Slovak Republic No. VEGA $1 / 0615 / 21$. 


\section{REFERENCES}

[1] OECD NEA, "International Handbook of Evaluated Critical Experiments," Paris, 2020.

[2] W. A. Wieselquist, et al., "SCALE Code System," ORNL/TM-2005/39, Version 6.2.4, Oak Ridge National Laboratory, Oak Ridge, TN, 2020.

[3] C. H. Warner, et al., "MCNP 6.2 Release Notes," LA-UR-18-20808, Los Alamos National Laboratory, Los Alamos, 2018.

[4] Š Čerba, et al., "Preliminary Results of the STU Mini Labyrinth Radiation Shielding Experiment," in Proc. APCOM 2021, St. Luis: AIP Publishing, 2018.

[5] Thermo Scientific, “ RadEye SPRD Personal Radiation Detector,” 2021, [Online]. Available: https://www.thermofisher.com/order/catalog/product/4250817\#/4250817 . Accessed on: April 6, 2021.

[6] R. V. Griffith, et. al., "Compendium of Neutron Spectra and Detector Responses for Radiation Protection Purposes, " Technical Report Series No. 318, IAEA, 1990.

[7] M. B. Chadwick, et. al, "ENDF/B-VII.0: Next Generation Evaluated Nuclear Data Library for Nuclear Science and Technology," Nucl. Data Sheets, vol. 107, pp. 2931-3060, Dec. 2006, DOI. 10.1016/j.nds.2006.11.001.

[8] M. B. Chadwick, et.al., "ENDF/B-VII.1 Nuclear Data for Science and Technology: Cross Sections, Covariances, Fission Product Yields, and Decay Data," Nucl. Data Sheets, vol. 112, pp. 2887-2996, Dec. 2011. DOI: 10.1016/j.nds.2011.11.002

[9] International Commission of Radiation Units and Measurements, "ICRU Report 57: Conversion Coefficients for Use in Radiological Protection Against External Radiation,” Bethesda, MD, Aug 1998.

[10] O. Sato, et. al., "Calculations of Effective Dose and Ambient Dose Equivalent Conversion Coefficients for High Energy Photons," J. Nucl. Sci. Technol., vol. 36, no. 11, pp. 977-987, Nov. 1999. DOI. 10.1080/18811248.1999.9726290

[11] W. Wieselquist, et. al., "Overview of Nuclear Data Uncertainty in Scale and Application to Light Water Reactor Uncertainty Analysis," NUREG/CR-7249, ORNL, USA, 117 pages., 2017.

[12] M. Herman, "Development of ENDF/B-VII.1 and its covariance component," BNL-93619-2010, BNL, USA, 6 pages, 2010. 